\title{
Erratum to: Combination Therapy of Transcatheter Arterial Chemoembolization and Arterial Administration of Antiangiogenesis on VX2 Liver Tumor
}

\author{
Gang Deng $\cdot$ Deng-Ling Zhao $\cdot$ Guang-Chao Li $\cdot$ \\ Hui Yu $\cdot$ Gao-Jun Teng
}

Published online: 15 September 2011

(C) Springer Science+Business Media, LLC and the Cardiovascular and Interventional Radiological Society of Europe (CIRSE) 2011

Erratum to: Cardiovasc Intervent Radiol (2011)

34:824-832

DOI 10.1007/s00270-011-0179-x

Readers should note that some unintended Chinese text characters were included in the published online and print article. Removing said text in the Discussion section results in the following corrected sentences.

\section{Paragraph 1:}

- These findings offer a rationale for combining mTOR inhibitors with chemotherapy in HCC treatment. Kan et al. [32] treated the implanted FN13762 murine mammary tumors in the ...

\section{Paragraph 6:}

- Although measurements of CT perfusion have been clinically demonstrated to be correlated with angiogenesis biomarkers, such as MVD in lung cancer and renal cancer [26], there are no such data available related to the liver tumor in the literature.

The online version of the original article can be found under doi:10.1007/s00270-011-0179-x.

G. Deng · D.-L. Zhao · G.-C. Li · H. Yu · G.-J. Teng ( $ه)$ Jiangsu Key Laboratory of Molecular Imaging and Function Imaging, Department of Radiology, Zhongda Hospital,

Medical School of Southeast University, 87\# Dingjiaqiao Road,

Nanjing 210009, China

e-mail: gjteng@vip.sina.com 